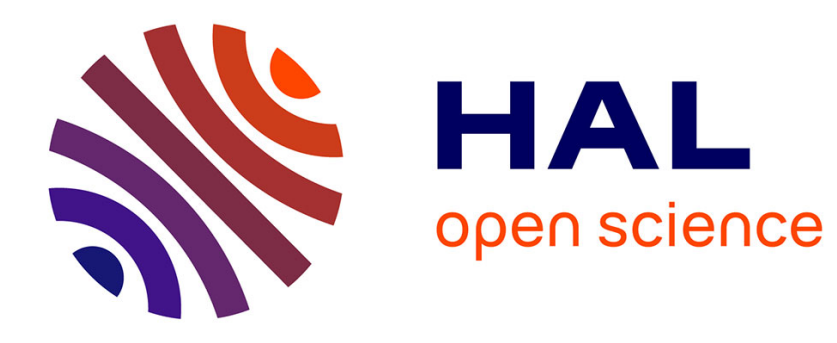

\title{
Phoxonic Crystals for Harnessing the Interaction of Light and Sound \\ Vincent Laude
}

\section{To cite this version:}

Vincent Laude. Phoxonic Crystals for Harnessing the Interaction of Light and Sound. International Conference on Optical MEMS and Nanophotonics, Jul 2016, Singapore, Singapore. 10.1109/OMN.2016.7565814 . hal-03053093

\section{HAL Id: hal-03053093 https://hal.science/hal-03053093}

Submitted on 10 Dec 2020

HAL is a multi-disciplinary open access archive for the deposit and dissemination of scientific research documents, whether they are published or not. The documents may come from teaching and research institutions in France or abroad, or from public or private research centers.
L'archive ouverte pluridisciplinaire HAL, est destinée au dépôt et à la diffusion de documents scientifiques de niveau recherche, publiés ou non, émanant des établissements d'enseignement et de recherche français ou étrangers, des laboratoires publics ou privés. 


\title{
Phoxonic Crystals for Harnessing the Interaction of Light and Sound
}

\author{
Vincent Laude \\ Institut FEMTO-ST, UMR CNRS 6174 \\ Université de Bourgogne Franche-Comté \\ Besançon, France
}

\begin{abstract}
Phoxonic crystals are periodic nanostructures that are simultaneously photonic and phononic crystals. Phoxonic cavities can trap visible or near infrared light and Gigahertz phonons in the same tiny volume, while phoxonic waveguides can confine their propagation to a tiny solid core, in both cases possibly promoting their interaction. This paper reviews various phoxonic nanostructures that have been proposed and discusses the interaction of light and sound within them.
\end{abstract}

Keywords-photonic crystal; phononic crystal; phoxonic crystal; photoelastic effect; moving interface effect.

\section{INTRODUCTION}

A phoxonic crystal designates a periodic structure that possesses simultaneously a photonic [1] and a phononic [2] band gap. The frequency band gap ranges for photons and phonons are usually vastly different. Given that the speed of elastic waves in solid materials is usually of the order of a few kilometers per second and that the speed of light in matter is of the order of a few $10^{5}$ kilometers per second, the ratio of the involved photonic and phononic frequencies is of the order of $10^{5}$. Yet the very idea of the phoxonic crystal is that the photon and phonon wavenumbers have comparable orders of magnitude, because of their connection with the period (or lattice constant) of the crystal. A consequence is that both the electromagnetic wave (for classically described photons) and the elastic wave (the continuum mechanical description of lowfrequency acoustic phonons) can be confined to the same tiny region of space of the order of the third power of the wavelength. This simultaneous confinement enables interactions between sound and light, or phonons and photons that renew the field of acousto-optics and opto-acoustics.

\section{TYPES OF PHOXONIC CRYSTALS}

\section{A. Bulk Phoxonic Crystals}

The concept of the phoxonic crystal was first introduced for the case of a nanostructure of silicon perforated with a periodical two-dimensional array of holes [3], following the discussion of the phononic properties of silica photonic crystal fibers [4]. These are both examples of bulk phoxonic crystals, meaning that waves propagate in an unbounded, though periodic, medium. Except for the case of the photonic crystal fiber, two-dimensional bulk phoxonic crystals are mostly a model for more complex structures. One important idea property is the existence of a complete phoxonic band gap, valid for all angles of incidence, or equivalently all wavevectors of reciprocal space [5]. One-dimensional phoxonic crystals have also been investigated, based on periodic multilayer stacks [6,7], though admittedly waves are not confined in the plane and thus only plane-wave operation can be achieved. Three-dimensional phoxonic crystals composed of metal spheres in an elastic/dielectric medium have also been proposed theoretically [8].

\section{B. Membrane Phoxonic Crystals}

Membrane-type phoxonic crystals, similar to photonic crystal slab structures, offer a practical basis for fabrication using standard nanotechnologies, e.g. based on silicon-overinsulator (SOI) wafers. In order to obtain a photonic band gap for the near-infrared, it is known that membrane thicknesses of the order of $250 \mathrm{~nm}$ and lattice constants of the order of 500 $\mathrm{nm}$ are required. With these orders of magnitude, phononic band gaps around 5 to $10 \mathrm{GHz}$ can be expected. The exploration of the optimal lattices and geometrical dimensions has been conducted in recent years for the case of circular holes [9,10], pillars [11], or mass-spring networks [12]. The first experimental demonstration of a phoxonic band gap was performed in the latter case [13].

\section{Design OF PhOXONIC CRYSTAL STRUCTURES}

\section{A. Holes, Pillars, or Stubs?}

The type of inclusion that should be considered to obtain complete and large phoxonic band gaps is not an obvious choice. Circular holes are a natural choice that is well compatible with nanotechnological processes. They are well suited for large photonic band gaps, but they require very large filling fractions, i.e. almost touching holes, for large phononic band gaps. Alternatively, large phononic band gaps can be obtained by combining Bragg band gaps with local resonances in the case of periodic arrays of 'masses' connected by 'springs'; masses are constituted from plain parts of the solid material while elastic springs are naturally formed by thin bridges etched in the same material. Pillars formed on top of the membrane also present natural local resonances that help form phononic band gaps. In addition, in the case of wire waveguides formed by structuration of the membrane, solid stubs can be added to the sides to act as local elastic resonators and combined with holes $[14,15]$. All in all, several types of working design solutions obtained by numerical optimization are now available [16]. 


\section{B. Cavities and Waveguides}

Various types of cavities and waveguides based on defects introduced in phoxonic crystals have been proposed. Those include Fabry-Perot interferometers in 1D phoxonic crystals [6,7], defect-based and tapered cavities in 2D phoxonic crystal slab structures $[13,17,18,19]$. Phoxonic crystal waveguides are also based on similar ideas and rely on a complete phoxonic band gap to ensure simultaneous light and sound confinement $[20,21,22]$.

\section{INTERACTION OF LIGHT AND SOUND}

The interaction of light and sound, or photons and phonons, in phoxonic crystals is governed by bulk and surface couplings. The coupling efficiency is usually rather small and interactions involve 2 photons (or 2 optical waves) and 1 phonon (or 1 elastic wave). The reason is that efficient interaction requires phase-matching conditions that can only be fulfilled by combining several frequencies in sums or differences. In the case of waveguides, in addition, wavevector matching must be achieved for efficient interaction, such as in the case of Brillouin light scattering by acoustic phonons.

\section{A. Photoelastic and Moving interface effects}

In acousto-optics, the dominant interaction occurs in the bulk of materials and is governed by an ad hoc photoelastic tensor. Multiplied by the strain tensor, which measures local deformations of the medium caused by the elastic wave, the photoelastic tensor gives the perturbative change in the electromagnetic permittivity. In phoxonic crystals, this photoelastic effect is obviously also present and is responsible for bulk inelastic light scattering.

Light confinement in phoxonic crystal relies on the strong contrast in dielectric constant between a material and air, or between different materials. As an elastic wave propagates, it causes a vibration of the interfaces and effectively modulates in time the resonance frequency of an optical cavity or the propagation constant (wavenumber) of an optical waveguide. This moving-interface effect can be significant in small core waveguides [23] and in silicon phoxonic crystal cavities $[18,19]$, for instance.

\section{B. Radiation Pressure and Electrostriction}

As light can be strongly confined in tiny waveguides or cavities, in addition to sensing high-frequency acoustic phonons it can generate them. In optomechanics, the basic generation mechanism is often attributed to radiation pressure that light exerts on dielectric interfaces and can set it into motion [23]. This generation effect of sound by light is the reciprocal of the moving interface effect involved in surface inelastic light scattering. In a similar way, bulk generation of sound by light can be attributed to the reciprocal of the photoelastic effect, or electrostriction [24]. All effects can be gathered in a unique Lagrangian formulation from which efficient finite element computations can be performed.

\section{REFERENCES}

[1] J.D. Joannopoulos, S.G. Johnson, J.N. Winn, and R.D. Meade, Photonic Crystals: Molding the Flow of Light, Princeton University Press, 2008.

[2] V. Laude, Phononic crystals, Berlin: De Gruyter, 2015.

[3] M. Maldovan and E.Thomas, "Simultaneous localization of photons and phonons in two-dimensional periodic structures," Appl. Phys. Lett., vol. 88, pp. 251907, 2006.

[4] V. Laude et al., "Phononic band-gap guidance of acoustic modes in photonic crystal fibers," Phys. Rev. B, vol. 71, pp. 045107, 2005.

[5] S. Sadat-Saleh, S. Benchabane, F.I. Baida, M.P. Bernal, and V. Laude, "Tailoring simultaneous photonic and phononic band gaps," J. Appl. Phys., vol. 106, pp. 074912, 2009.

[6] M. Trigo, A. Bruchhausen, A. Fainstein, B. Jusserand, and V. ThierryMieg, "Confinement of acoustical vibrations in a semiconductor planar phonon cavity," Phys. Rev. Lett., vol. 89, pp. 227402, 2002.

[7] I.E. Psarobas et al., 'Enhanced acousto-optic interactions in a onedimensional phoxonic cavity," Phys. Rev. B, vol. 82, pp. 174303, 2010.

[8] N. Papanikolaou, I.E. Psarobas, and N. Stefanou, "Absolute spectral gaps for infrared light and hypersound in three-dimensional metallodielectric phoxonic crystals," Appl. Phys. Lett., vol. 96, pp. 231917, 2010 .

[9] S. Mohammadi, A.A. Eftekhar, A. Khelif, A. Adibi, "Simultaneous twodimensional phononic and photonic band gaps in optomechanical crystal slabs," Opt. Express, vol. 18, pp. 9164-72, 2010.

[10] Y. Pennec et al., "Simultaneous existence of phononic and photonic band gaps in periodic crystal slabs," Opt. Express, vol. 18, pp. 1430110, 2010.

[11] Y. El Hassouani et al., "Dual phononic and photonic band gaps in a periodic array of pillars deposited on a thin plate," Phys. Rev. B, vol. 82, pp. 155405, 2010

[12] T.X. Ma, Y.S. Wang, Y.F. Wang, and X.X. Su., "Three-dimensional dielectric phoxonic crystals with network topology," Opt. Express, vol. 21, pp. 2727-32, 2013.

[13] A.H. Safavi-Naeini, J.T. Hill, S. Meenehan, J. Chan, S. Groeblacher, and O. Painter, "Two-dimensional phononic-photonic bandgap optomechanical crystal cavity," Phys. Rev. Lett., vol. 112, pp. 153603, 2014.

[14] Y. Pennec et al., "Band gaps and cavity modes in dual phononic and photonic strip waveguides,” AIP Advances, vol. 1, pp. 041901, 2011.

[15] J. Gomis-Bresco et al., "A 1D Optomechanical crystal with a complete phononic band gap," Nat. Commun., vol. 5, pp. 4452, 2014.

[16] H.W. Dong, Y.S. Wang, T.X. Ma, and X.X. Su, «Topology optimization of simultaneous photonic and phononic bandgaps and highly effective phoxonic cavity," JOSA B, vol. 31, pp. 2946-2955, 2014.

[17] Q. Rolland et al., "Acousto-optic couplings in two-dimensional phoxonic crystal cavities," Appl. Phys. Lett. Vol. 101, pp. 061109, 2012.

[18] S. El-Jallal et al., "Analysis of optomechanical coupling in twodimensional square lattice phoxonic crystal slab cavities," Phys. Rev. B, vol. 88, pp. 205410, 2013.

[19] Y. Pennec et al. "Modeling light-sound interaction in nanoscale cavities and waveguides," Nanophotonics, vol. 3, pp. 413-440, 2014.

[20] V. Laude et al., "Simultaneous guidance of slow photons and slow acoustic phonons in silicon phoxonic crystal slabs," Opt. Express, vol. 19, pp. 9690-8, 2011.

[21] J.M. Escalante, A. Martínez, and V. Laude, "Design of single-mode waveguides for enhanced light-sound interaction in honeycomb-lattice silicon slab," J. Appl. Phys., vol. 115, pp. 064302, 2014.

[22] K.C. Balram, M.I. Davanço, J.D. Song, and K. Srinivasan, "Coherent coupling between radiofrequency, optical and acoustic waves in piezooptomechanical circuits," Nat. Phot., vol. 10, pp. 346-352, 2016.

[23] P.T. Rakich, C. Reinke, R. Camacho, P. Davids, and Z. Wang., "Giant Enhancement of stimulated brillouin scattering in the subwavelength limit," Phys. Rev. X, vol. 2, pp. 011008, 2012.

[24] V. Laude and J.C. Beugnot, "Lagrangian description of Brillouin scattering and electrostriction in nanoscale optical waveguides," New J. Phys., vol. 17, pp. 125003, 2015. 